\title{
Photonically Enabled Communication Systems Beyond 1000 GHz (Invited Paper)
}

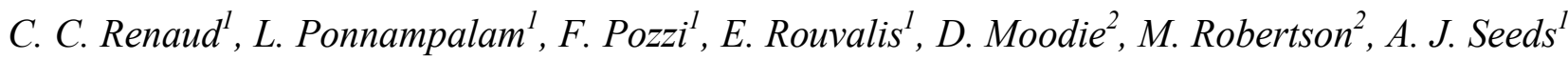 \\ ${ }^{1}$ Department of Electronic and Electrical Engineering, University College London, Torrington \\ Place, London, WC1E 7JE, United Kingdom \\ ${ }^{2}$ Centre for Integrated Photonics, Ipswich, IP5 3RE, United Kingdom
}

\begin{abstract}
This paper presents a review of the recent development and research work on InP devices and their associated systems to generate and detect signal beyond $1 \mathrm{THz}$. The potential of the technology and the remaining challenges are also discussed. The paper will present recent results on laser sources that could be used as the basis of the THz sources as well as a set of potential THz emitters such as the UTC photodiode which has already permitted up to $25 \mu \mathrm{W}$ to be emitted at $1 \mathrm{THz}$.
\end{abstract}

Index Terms - THz, Photonic system, photodetectrors, Optical frequency comb generation, Laser diode, FM laser.

\section{INTRODUCTION}

$\mathrm{THz}$ signals (Usually considered as signals of frequency from $100 \mathrm{GHz}$ to $10 \mathrm{THz}$ ) are attracting more and more interest as they offer potential for applications such as ultra-broadband wireless communication (lower $\mathrm{THz}$ range due to water absorption limiting the range), security imaging, gas sensing, molecular spectroscopy, non destructive monitoring and many others. One of the issues of the $\mathrm{THz}$ range is the limited number of sources to generate such signals. So far the main approach for $\mathrm{THz}$ systems is to use short-pulses lasers, which can offer signal ranging from a few GHZ to several $\mathrm{THz}$ [1], or for the lower range (below 1THz) the use of Gunn diodes [2]. Direct optical sources such as the quantum cascade lasers, which can offer signal from $1.3 \mathrm{THz}$ up to $10 \mathrm{THz}$ [3] with $\mathrm{CW}$ power up to $15 \mathrm{~mW}$ at $2 \mathrm{THz}$. However such devices are not easily tuneable (limiting potential applications) and in the lower frequency range they require liquid helium cooling.

This paper discusses the potential of using photonic based systems with InP technology for signals above $1000 \mathrm{GHz}$ and their potential for creating a compact $\mathrm{THz}$ system. In particular, the work done at UCL on monolithically integrated comb generators, high purity integrated heterodyne sources, and high-speed photodetectors will be described. In more detail, the first part will demonstrate the different advantages of heterodyne systems and show how they allow the generation of high purity signals at frequencies beyond $1 \mathrm{THz}$. In this part the advances in InP based technology to create such heterodyne sources, such as monolithic comb generators and the development of integrated optical phase locked loops, will also be discussed. The second part will describe the different potential emitters based on InP technology and detail the results obtained with uni-travelling carrier (UTC) photodetectors. Finally the problem of $\mathrm{THz}$ detection and coherent systems based on the same optical communication technology will be discussed and some potential solutions described, including optically pumped UTC mixers.

\section{HETERODYNE SOURCES}

The heterodyne source, as shown in Figure 1, comprises three main sub-systems, two of which will be discussed in this part and the third in part III. The first sub-system is the master laser, which is chosen to be an optical frequency comb generator. This offers the advantages of providing a reference source locked to a microwave synthesizer that will determine the overall spectral purity of the generated signal. Several solutions have emerged for such sources such as deep angle modulation of an optical source [4] to provide lines over a span of up to 4 $\mathrm{THz}$, or mode-locked semiconductor lasers [5]. Both can provide equally spaced lines over a wide span, however the first solution only provides small power in each lines while the second one creates deep intensity modulation leading to saturation issues in the second sub-system. The two chosen solutions for the heterodyne $\mathrm{THz}$ generator were either an amplified fiber loop comb generator or a recently developed monolithic FM laser [6] as shown in Figure 2. This laser is a single epitaxial growth device comprising three sections providing gain, phase adjustment and phase modulation. The fabrication of the FM laser was made possible by combining two fabrication technologies for InP devices. First, quantum

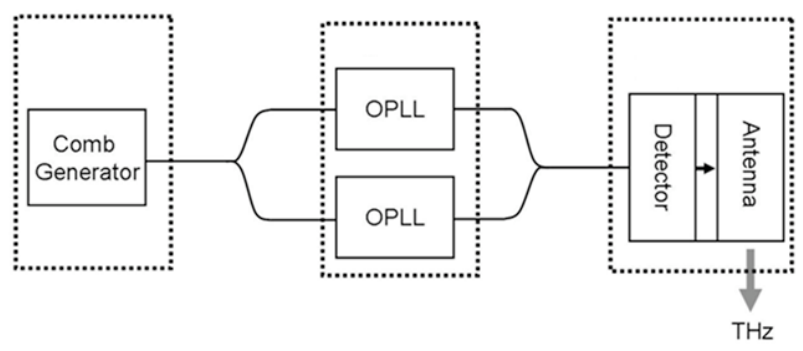

Figure 1: Schematic of the heterodyne system 

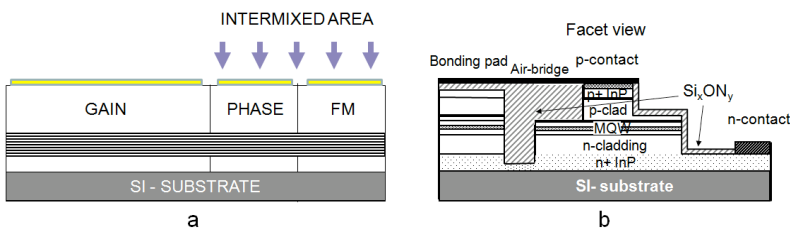

Figure 2: Schematic of the monolithic FM laser

well intermixing [7] was applied in order to shift the bandgap of the phase and modulation section to reduce signal absorption. Then oxide-bridge contacts were fabricated to obtain a low capacitance modulation section to allow for modulation speeds up to $25 \mathrm{GHz}$. These modulation speeds were obtained by using refractive index modulation due to the quantum confined Stark effect [8] needing reverse bias modulation and giving a response that is not limited by carrier transport. This solution offers the advantage of low intensity modulation (less than 20\%) and the generation of lines spaced by the microwave reference source frequency. Figure 3 shows the measured heterodyne of two adjacent lines of the FM laser compared with the phase noise due to the reference source. The difference of noise floor is only due to the difference of peak intensity of the two measured signals ( $10 \mathrm{dBm}$ for the source and $-10 \mathrm{dBm}$ for the heterodyne). Apart from that difference this measurement demonstrate that the quality of the comb line heterodyne is limited by the reference microwave source.

The second sub-system comprises two optical phase locked loop (OPLL) laser filters that will generate the heterodyne signal. OPLL filters are chosen to lock the slave lasers to lines of the master OFCG. OPLL filters are chosen for their wide locking bandwidth compared to optical injection locked filters. However, one of the issues of OPLL filters is that in order to lock a standard semiconductor laser with a linewidth of $1 \mathrm{MHz}$ the loop delay should be shorter than $1 \mathrm{~ns}$ [9]. To overcome this problem two solutions are possible. One is to use an optical injection phase locked loop (OIPLL), which retains some advantages from the OPLL (long term

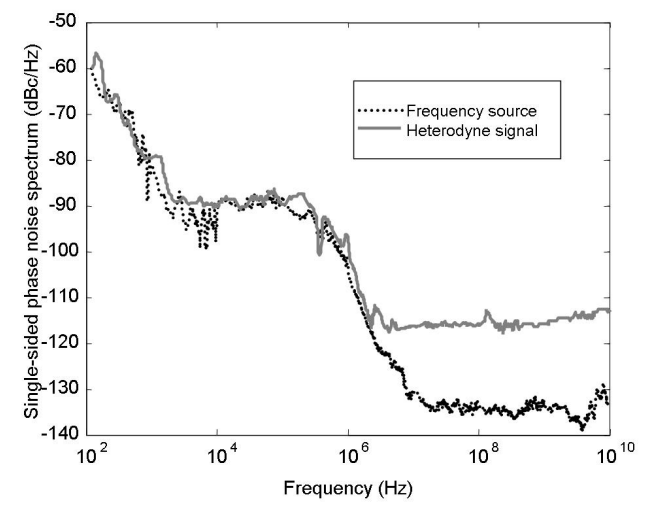

Figure 3: Phase noise measurement of the microwave reference source and the heterodyne of two adjacent comb lines.

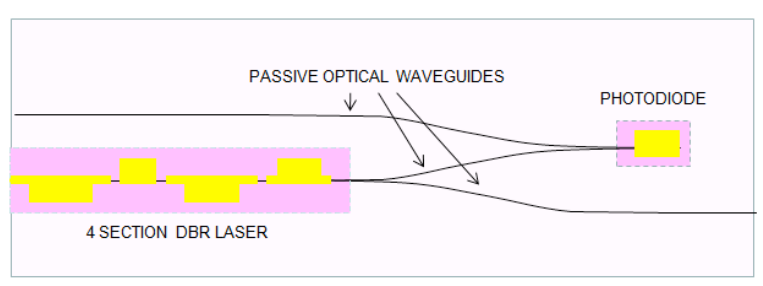

Figure 4: Schematic of the monolithically integrated OPLL

tracking) but the short loop is not needed thanks to the optical injection. However this limits the frequencies obtainable with the heterodyne system to steps determined by the comb generator. The other solution is to integrate the OPLL in order to reduce the delay. The preferred solution would be a monolithic integration of the optical part of the OPLL (Figure 4) where the various parts of the single optical OPLL are defined on the same chip. These elements are a four section distributed Bragg reflector laser, passive waveguides including couplers, and a photodiode. The laser is designed to provide a wavelength tuning range of approximately $8 \mathrm{~nm}$, so that two lasers with an offset of $7 \mathrm{~nm}$ could offer heterodyne signals ranging from 0 to $2 \mathrm{THz}$. The tuning sections and the passive waveguides are fabricated using selective area regrowth technique. However as the detector needs to detect frequencies up to $10 \mathrm{GHz}$ it is designed as a ridge waveguide detector in order to reduce the device capacitance. Such a design offers the further possibility of integrating the two lasers on the same ship, thus allowing close thermal tracking of the lasers and easing requirements on the control loop.

The advantage of this heterodyne system for $\mathrm{THz}$ signal generation is demonstrated by the measured heterodyne signal at $110 \mathrm{GHz}$ shown in Figure 5. This experiment demonstrated that the quality of the microwave source driving the comb generator was retained in the final heterodyne signal. This signal full half width maximum is less than $10 \mathrm{~Hz}$ thus it is clear that this could be an ideal source to generate high purity signals from $1 \mathrm{THz}$ upward.

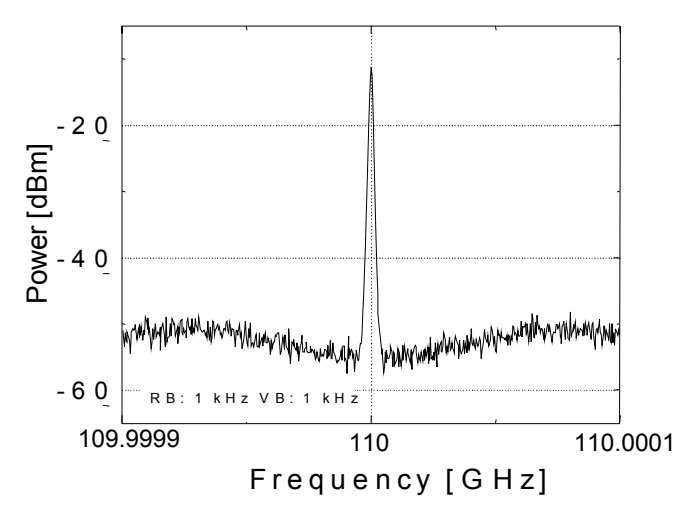

Figure 5: High purity heterodyne signal at $110 \mathrm{GHz}$ 


\section{THz EMITTERS}

Although the optical heterodyne signal can be of high purity and reach frequencies well above $1 \mathrm{THz}$, the main limitation of the system will be the $\mathrm{THz}$ emitter. In order to be able to use optical communications components working at $1.5 \mu \mathrm{m}$ wavelength, two main InP based solutions have appeared, they are either the use of InP based photoconductors where the recovery time can be made faster using a set of different techniques [10], or the use of fast photodetectors (PD) such as the traveling wave (TW) PD [11] or uni-travelling carrier (UTC) PD [12]. The latter will ultimately be limited in the $\mathrm{THz}$ range, however recent developments have shown that emissions at the $\mu \mathrm{W}$ level are possible around $1 \mathrm{THz}$ [13]. The chosen solution for our work is a development of the UTC photodetector using traveling wave techniques. The main advantages of using the UTC structure are the faster drift velocity of the electrons and the reduction of the carrier space charge effects that should allow for higher saturation powers. Furthermore designing the device as a pseudo-TW will enhance the power extraction at higher frequencies as well as the saturation performance. The UTC structure used for this work was designed to offer a short absorption length to reduce the parasitic capacitance thus limiting the effect of the traveling wave design. This resulted in an absorption layer thickness such that the carrier transit limited $3 \mathrm{~dB}$ bandwidth was about $340 \mathrm{GHz}$. The length of the waveguide device necessary to absorb $90 \%$ of the incoming light was calculated to be $15 \mu \mathrm{m}$. Once fabricated the $15 \mu \mathrm{m}$ long waveguide device was measured to have a parasitic capacitance of about $25 \mathrm{fF}$, which gives a capacitance limited $3 \mathrm{~dB}$ bandwidth of 127 $\mathrm{GHz}$ with a $50 \Omega$ load, which should be our main limitation on the bandwidth of the detector. The detector was integrated with either a broadband bow-tie antenna (design for emission ranging from about $600 \mathrm{GHz}$ to above $2 \mathrm{THz}$ ) or a resonant antenna (at $457 \mathrm{GHz}$ ). Both devices were tested using the heterodyne system followed by an erbium-doped amplifier for a measured photocurrent of $10 \mathrm{~mA}$. In the case of the resonant antenna the extracted power was measured using a THZ photo-acoustic power meter. It was $148 \mu \mathrm{W}$ at $457 \mathrm{GHz}$ and at the harmonic frequency of $914 \mathrm{GHz}$ the power was $24 \mu \mathrm{W}$. The device with the bow-tie antenna did not

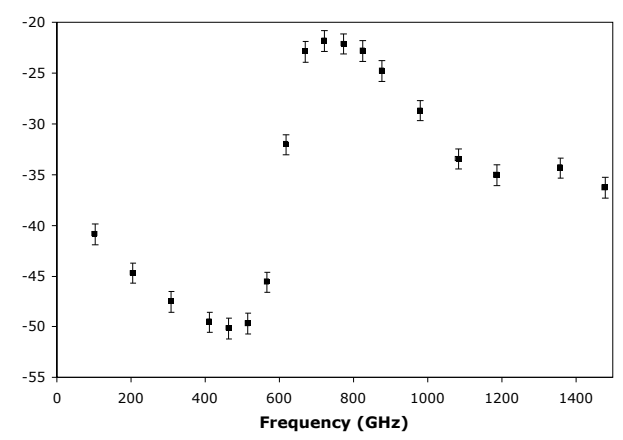

Figure 6: Measured radiation from the TW-UTC device integrated with a bow-tie antenna

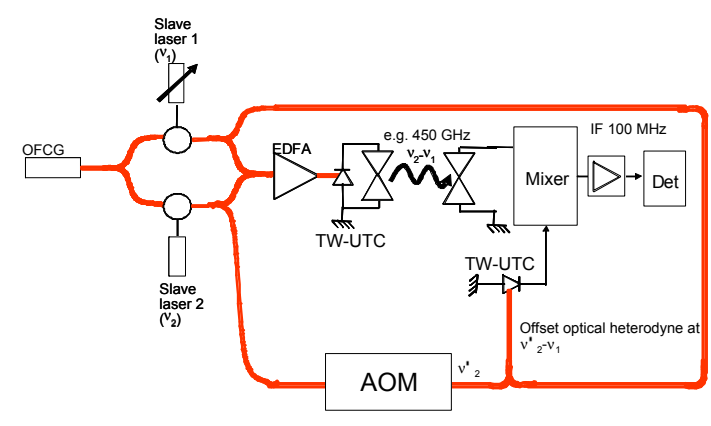

Figure 7: Schematic of the coherent heterodyne $\mathrm{THz}$ system including detection.

achieve such high powers and a more sensitive Golay cell was used to measured the extracted power for frequencies up to $1.5 \mathrm{THz}$ (limited by the OFCG of the heterodyne system). The resulting extracted power is shown in Figure 6 and the device achieved powers ranging from $0.2 \mu \mathrm{W}$ at $1.5 \mathrm{THz}$ to a maximum of $6 \mu \mathrm{W}$ at $720 \mathrm{GHz}$. Note that this result is dependent on the Golay cell calibration which was done at $110 \mathrm{GHz}$ using horn antennas and a calibrated power meter for comparison.

\section{THz DETECTION}

The key to have a full system beyond $1000 \mathrm{GHz}$ is a suitable detection system. In that case there is an advantage to adapt coherent systems to $\mathrm{THz}$ detection. One proposed approach is to develop an homodyne system [14], which is also based on an heterodyne source with one of the branches carrying the path adjustment necessary for matching the phase of the emission and the detection. The other approach, which we have chosen is based directly on our high spectral purity heterodyne system. The full coherent system is shown in Figure 7. In such a system the signal is generated as described earlier by heterodyning two lasers locked to a master source and then emitted through an antenna connected to a fast optoelectronic device such as the TW-UTC. The signal is collected through an antenna while another heterodyne signal with a slight frequency shift from the other (Provided by an acousto-optic modulator in Figure 7) is sent to the TW-UTC to be used as a local oscillator. The signal collected from the antenna is then mixed with the local oscillator heterodyne signal from the TW-UTC and the difference of the two frequencies (the intermediate frequency) is then measured using conventional electronic systems. As the power sensitivity of such a system is dependent on the measurement bandwidth, the high purity of the heterodyne signal becomes important. For example a standard heterodyne system based on semiconductor lasers will offer a linewidth in the $\mathrm{MHz}$ range while the system we have developed can offer a linewidth of less than $10 \mathrm{~Hz}$. As the linewidth of the heterodyne signal will set the limit to the detection bandwidth it is obvious that such a system could offer up to 5 orders of magnitude improvement in term of measurement sensitivity. However this improvement will 


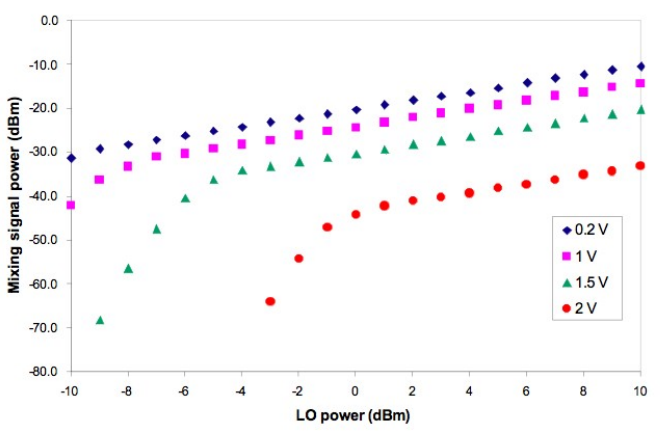

Figure 8: Measured intermediate frequency power as a function of the optical Local oscillator.

be largely dependent on the room temperature mixing technology to be developed. One potential solution could be the use of Schottky diodes, which have proved efficient in that range of frequency [15]. Another proposed solution is to use UTC structure as optoelectronic mixers where the fast carrier transport and non-linearities should allow for mixing of an optical heterodyne local oscillator with an electric signal received by an antenna. A proof of concept demonstration of optoelectronic mixing was made with the UTC detector developed for this work at $10 \mathrm{GHz}$. The results are shown in Figure 8. As it can be seen, for a bias of $1.5 \mathrm{~V}$, where the device has a good frequency response, the conversion losses are about $30 \mathrm{~dB}$ when the local oscillator (Optical) is about $8 \mathrm{dBm}$. These results encourage us to believe that such devices are a potential option for the $\mathrm{THz}$ receiver in the system.

\section{CONCLUSION}

This work demonstrates the potential of optical communication based technology for the development of systems working above $1000 \mathrm{GHz}$. In particular the development of InP based emitters working at $1.5 \mu \mathrm{m}$ offering the possibility of generating signals above $1000 \mathrm{GHz}$ with output power of more than $0.1 \mu \mathrm{W}$. Furthermore, the development of monolithic devices to provide the OFCG, and the OPLL filters shows the potential of the technology to enable portable $\mathrm{THz}$ system with frequencies up to $2 \mathrm{THz}$. We have demonstrated FM operation in a monolithic semiconductor laser. This optical frequency combgenerator offers $24.4 \mathrm{GHz}$ spaced lines over a spectral width of $15 \mathrm{~nm}(\sim 2 \mathrm{THz})$. The narrow linewidth of the resulting signal allow for a potential five orders of magnitude gain in detection sensitivity if the technology is combined into a heterodyne coherent detection system. It is also important to note that the compactness of the different elements of the system should allow for the development of array systems that will improve the output power of the $\mathrm{THz}$ source, thus creating a more efficient system. Furthermore modulation could be applied to the heterodyne signal (not on the local oscillator), and should be detected on the intermediate frequency, thus allowing transport of information on the heterodyne signal. Being based on communications technology this system could offer a cost effective solution for applications needing frequencies in the range $100 \mathrm{GHz}$ to $2000 \mathrm{GHz}$.

\section{ACKNOWLEDGEMENT}

This work is supported by the European Union under the Integrated project IPHOBAC and by the Engineering and Physical Science Research Council under the projects PORTRAIT and PHITSIN.

\section{REFERENCES}

[1] A. J. Lucero Y. C. Chung, R. W. Tkach, IEEE Photon. Technol. Lett., vol. 3, pp.484-86, 1991

[2] S. K. Panigrahi, A. K. Panda, Proceedings of APSYM 2002. National Symposium on Antennas \& Propagation, 2002, p 193-6

[3] C. Walther, G. Scalari, J. Faist, H. Beere and D. Ritchie, "Low frequency terahertz quantum cascade laser operating from 1.6 to $1.8 \mathrm{THz}, "$ Applied Physics Lett., vol. 89, pp. 231121-23, 2006.

[4] M. Kourogi, K. Nakagawa, M. Ohtsu, IEEE J. Quantum Electron, Vol. 29, pp. 2693-701, 1993.

[5] F. van Dijk, A. Enard, X. Buet, F. Lelarge and G.H. Duan, IEEE International Topical Meeting on Microwave Photonics, 2007, pp. 66-69.

[6] C. C. Renaud, M. Pantouvaki, S. Gregoire, I. Lealman, P. Cannard, S. Cole, R. Moore, R. Gwilliam and A. Seeds, IEEE J. of Quantum Electron., vol. 43, pp. 998-1005, 2007.

[7] J.H. Marsh and A.C. Bryce, Optoelectronic properties of semiconductor and superlattices, 1999, pp. 33970.

[8] B. Cai, A. J. Seeds, J. S. Roberts, IEEE Photon. Technol. Lett., Vol. 6, pp. 496-98, 1994

[9] R.T. Ramos and A.J. Seeds, Electron. Lett., vol. 26, pp. 389-91, 1990.

[10] J. Mangeney, L. Joulaud, N. Chimof, P. Crozat, C. Boukari, M. Riet, R. Levevre, IPRM 2005, 2005, pp. 586-9.

[11] A. Stöhr, R. Heinzelmann, K. Hagedorn, R. Gusten, F. Schafer, H. Stuer, F. Siebe, P. van der Wal, V. Krozer, M. Feiginov, D. Jager, Electron. Lett., vol. 37, pp. 1347-48, 2001

[12] H. Ito, S. Kodama, Y. Muramoto, T. Furuta, T. Nagatsuma, T. Ishibashi IEEE J. of Selected Topics in Quantum Electron, vol. 10, pp 709-27, 2004.

[13] C.C. Renaud, M. Robertson, D. Rogers, R. Firth, P.J. Cannard, R. Moore and A.J. Seeds, Proceedings of SPIE Photonics Europe, 2006, pp. 61940C-1$61940 \mathrm{C}-8$.

[14]R. Wilk, M. Mikulics, K. Biermann, H. Kunzel, I. Z. Kozma, R. Holzwarth, B. Sartorius, B. Mei, M. Koch, CLEO '07. 2007 Conference on Lasers and Electro-Optics, 2007, p 1033-4

[15]U. R. Pfeiffer, C. Mishra, Member, R. M. Rassel, S. Pinkett, S. K. Reynolds, IEEE Trans. On microwave Theory and Technique, Vol 56, pp. 364-371, 2008 